\title{
Pioderma Gangrenoso Associado a Hemoglobinúria Paroxística Noturna
}

\author{
Francisco Ronaldo Moura Filho', MD, Aline Guimarães Grana', MD, Suzi Marla Carvalho Maron', MD, Raquel Rodrigues Ferreira \\ Rocha de Alencar ${ }^{2}$, Bruno Vianei Real Antonio ${ }^{2}$, Patricia Chicre Bandeira de Melo ${ }^{3}$, MD, MSc. \\ 'Médico Residente/Resident of Dermatology, Serviço de Dermatologia do Hospital Universitário Getúlio Vargas (HUGV), \\ Universidade Federal do Amazonas (UFAM), Manaus, Brasil. \\ ${ }^{2}$ Acadêmico de Medicina/Medical Academic, da Universidade Federal do Amazonas, Manaus, Brasil \\ ${ }^{3}$ Dermatologista Especialista pela Sociedade Brasileira de Dermatologia/Dermatologist, Mestre em Medicina Tropical pela \\ Universidade do Estado do Amazonas, Manaus, Brasil
}

RESUMO - O pioderma gangrenoso é uma dermatose neutrofílica crônica sem etiologia estabelecida. Sabe-se que se associa a doenças sistémicas como doenças reumatológicas, doenças inflamatórias intestinais, neoplasias malignas, doenças hematológicas, dentre elas a hemoglobinúria paroxística noturna. Manifesta-se através de lesões cutâneas ulceradas e dolorosas com evolução rápida e progressiva, sendo mais comum nos membros inferiores. As ulcerações podem surgir espontaneamente ou induzidas por traumas, sendo característico do pioderma gangrenoso o fenômeno de patergia. O período entre o início das lesões e o diagnóstico costuma ser prolongado. O diagnóstico é baseado em evidências clínicas e confirmado com a exclusão das outras etiologias de lesões ulceradas cutâneas. Relatamos um caso de pioderma gangrenoso em membro superior esquerdo com início após evento traumático em paciente com diagnóstico de hemoglobinúria paroxística noturna.

PALAVRAS-CHAVE - Hemoglobinúria Paroxística; Pioderma Gangrenoso.

\section{Pyoderma Gangrenosum associated with Paroxysmal Nocturnal Haemoglobinuria}

ABSTRACT - Pyoderma gangrenosum is a chronic neutrophilic dermatosis with no established etiology. It is often associated with systemic diseases such as inflammatory rheumatic diseases, inflammatory bowel diseases, malignant neoplasms and haematological diseases including nocturnal paroxysmal hemoglobinuria. It presents as ulcerated and painful skin lesions with rapid and progressive evolution, mainly in the lower limbs. Ulcerations may arise spontaneously or induced by trauma (pathergy phenomenon). The period between the onset of the lesions and the diagnosis is usually prolonged. The diagnosis is based on clinical data and confirmed after the exclusion of other causes of cutaneous ulcerations. We report a case of Pyoderma gangrenosum in the left upper limb with onset after a traumatic event in a patient with nocturnal paroxysmal hemoglobinuria.

KEYWORDS - Hemoglobinuria, Paroxysmal; Pyoderma Gangrenosum.

\section{INTRODUÇÃO}

O pioderma gangrenoso (PG) é uma dermatose inflamatória neutrofílica, não infeciosa, rara de evolução crónica e recidivante, que afeta sobretudo mulheres da faixa etária entre os 25 e 54 anos. ${ }^{1,2}$ Apesar dos avanços científicos sobre o entendimento do PG, sua etiologia não é bem estabelecida. As evidências de distúrbios em células imunes são muito convincentes, mas insuficientes para explicar totalmente a patogênese desta doença. ${ }^{2}$ Existem relatos na literatura associando o PG ao fenómeno de patergia, ou seja, o surgimento de novas lesões desencadeado por traumas, incluindo cirúrgicos. ${ }^{1,3,4}$

Acredita-se que o esclarecimento sobre a etiologia do
Correspondência: Francisco Ronaldo Moura Filho

Rua Ramos Ferreira, 199, Aparecida - CEP: 69010120, Manaus, AM, Brazil

Tel.: +5585997366000 / +5592981076000

E-mail: fronaldomoura@hotmail.com
Recebido/Received

29 Outubro/October 2016

Aceite/Accepted

19 Janeiro/January 2017 


\section{Caso Clínico}

PG esteja presente na associação com doenças sistêmicas (doenças reumatológicas, doenças inflamatórias intestinais, neoplasias malignas, doenças hematológicas dentre elas a hemoglobinúria paroxística noturna), ,-8 uma vez que tal relação está presente em 50 a 70\% dos casos. ${ }^{2,7}$ O PG também pode ser considerado idiopático, apresentando-se como uma lesão primária, isolada e restrita à pele. ${ }^{2}$

Neste trabalho é apresentado um caso de PG com ótima resposta à corticoterapia em paciente portadora de hemoglobinúria paroxística noturna (HPN), uma associação bastante rara.

\section{RELATO DE CASO}

Paciente do sexo feminino, 58 anos, portadora de diabetes mellitus tipo 2 há quatorze anos e HPN há um ano tratada com sulfato ferroso $80 \mathrm{mg} /$ dia de uso contínuo. Relata arranhadura no antebraço esquerdo que oito dias depois, evoluiu com eritema, bolhas, exulceração e dor importante. Avaliada no Serviço de Urgência, foi prescrito antibioticoterapia (cefalexina e penicilina benzatina) não obtendo melhora. Treze dias após início do quadro, a doente ficou internada devido ao agravamento das lesões cutâneas.

Ao exame físico, foi verificada placa eritemato-violácea única e extensa em todo antebraço esquerdo, que se expandia para o dorso da mão, com áreas exulceradas, algumas encimadas por crostas, e bordos descolados até a região das articulações metacarpofalangianas e pregas interdigitais (Fig.s 1 e 2). Foram colocadas as hipóteses de pioderma gangrenoso, micobacteriose atípica e vasculite. A paciente recebeu novo tratamento antibiótico e realizou biópsia da lesão, exames laboratoriais e de imagem para melhor esclarecimento diagnóstico.

Dentre os exames laboratoriais, foram vistas alterações no hemograma, com hemoglobina variando entre 7,7 e 9,5 g/ $\mathrm{dL}$, sendo o valor de referência(VR): 12 a $16 \mathrm{~g} / \mathrm{dL}$ ), hematócrito entre 20,7 e 29,3\% (VR: 35 a 47\%), red cell distribution

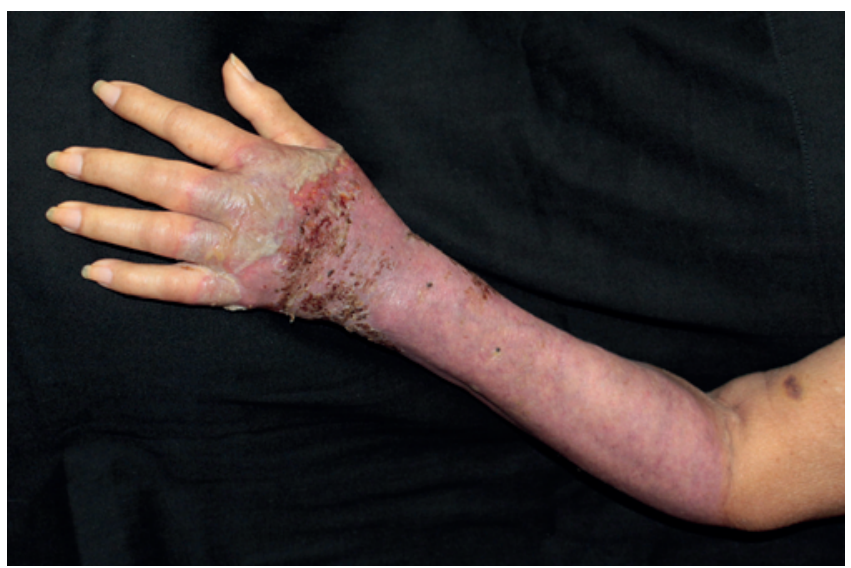

Figura 1 - Placa eritemato-violácea única e extensa em todo ante braço esquerdo, a qual expandiu para dorso da mão com áreas exulceradas, algumas encimadas por crostas, descolamento até a região das articulações metacarpofalangianas e pregas interdigitais.

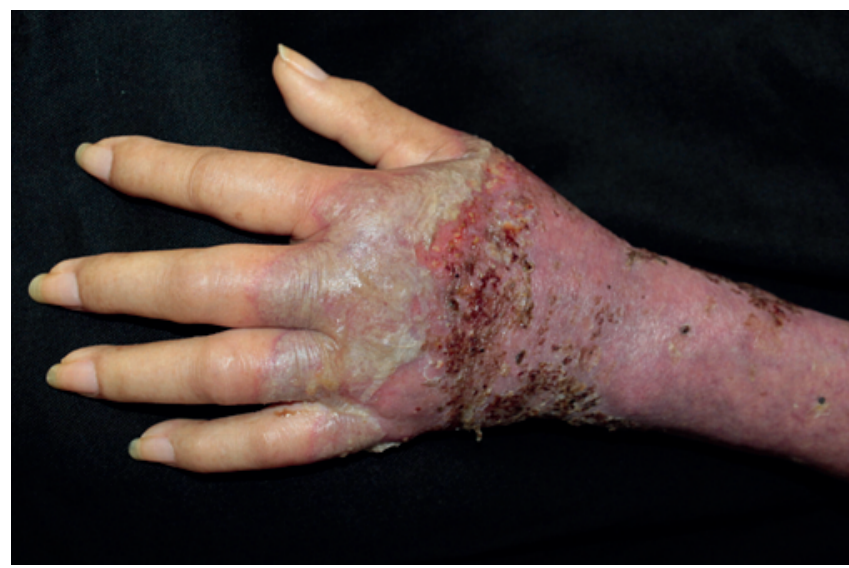

Figura 2 - Pormenor da placa eritematosa no dorso da mão, com evidência do bordo pustuloso descolado a nível das articulações metacarpofalangianas e pregas interdigitais.

width (RDW) entre 16 e 18,3\% (VR: 11 a 16\%), leucócitos entre 4210 e 10060 (VR: 5000 a 10 000), neutrófilos entre 66 e $78 \%$ (VR: 40 a 75\%), plaquetas entre 198000 a 368 000 (VR: 150000 a 450 000), e ainda glicémia entre 147 e $203 \mathrm{mg} / \mathrm{dL}$ (VR: 70 a $100 \mathrm{mg} / \mathrm{dL}$ ), proteína C reativa (PCR) entre 3,63 e 7,9 (VR: menor que 0,5 mg/dL), velocidade de

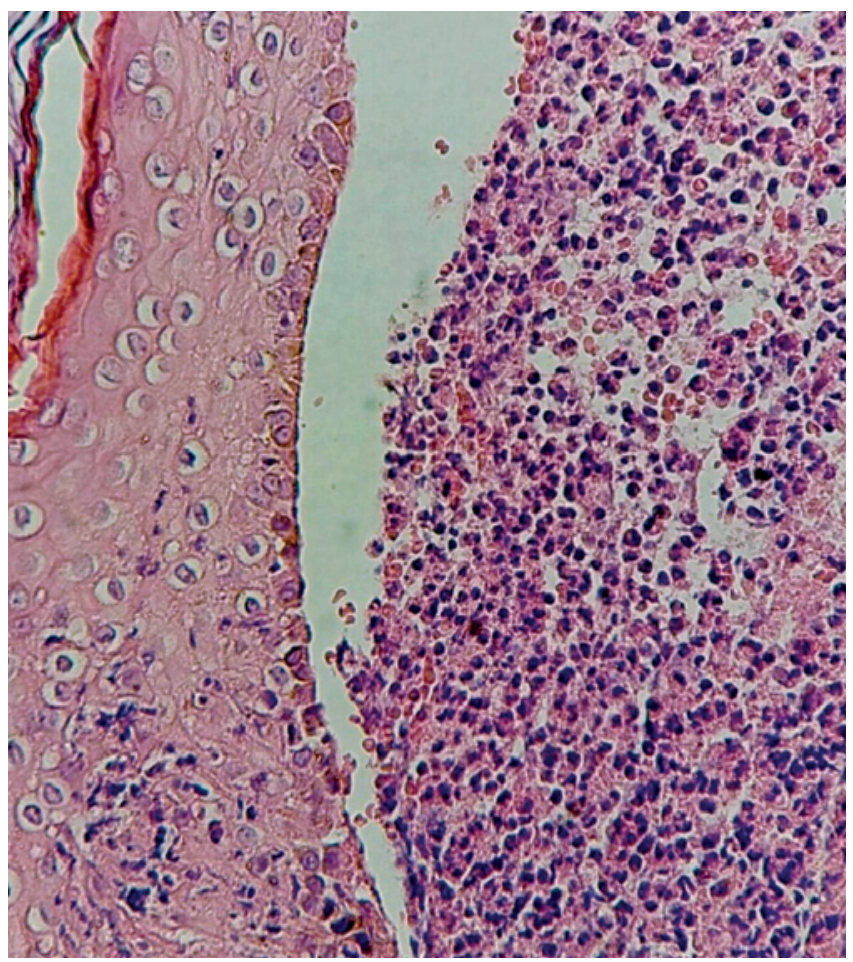

Figura 3 - Descolamento dermoepidérmico. Na epiderme observam-se queratinócitos apoptóticos e discreta espongiose e exocitose de neutrófilos. Na derme observa-se um infiltrado inflamatório importante e difuso, constituído predominantemente por neutrófilos, observando-se áreas com leucocitoclasia, edema e hemorragia. 


\section{Caso Clínico}

hemossedimentação (VHS) entre 125 e 171 mm (VR: até 20 $\mathrm{mm}$ ) e bilirrubina indireta 0,23 $\mathrm{mg} / \mathrm{dL}(V R:<0,7 \mathrm{mg} / \mathrm{dL})$ e ferro sérico ( $\mathrm{Fe}$ ) entre 20 e $80 \mathrm{mcg} / \mathrm{dL}$ (VR: 60 a $180 \mathrm{mcg} / \mathrm{dL}$ )

O exame histopatológico mostrou epiderme com alguns queratinócitos apoptóticos e moderada espongiose e exocitose, além de descolamento dermoepidérmico. Na derme superficial observou-se um infiltrado inflamatório difuso e denso, predominantemente constituído por neutrófilos, edema e presença de leucocitoclasia. $O$ infiltrado neutrofílico estendia-se à derme média e profunda. Na restante derme existia infiltrado inflamatório com predomínio neutrofilico em redor de fragmentos de anexos cutâneos e extensa necrose do colágeno (Fig.s 3 e 4). Face à correlação clínico-patológica e exclusão das outras hipóteses diagnósticas, foi instituída terapia imunossupressora para PG.

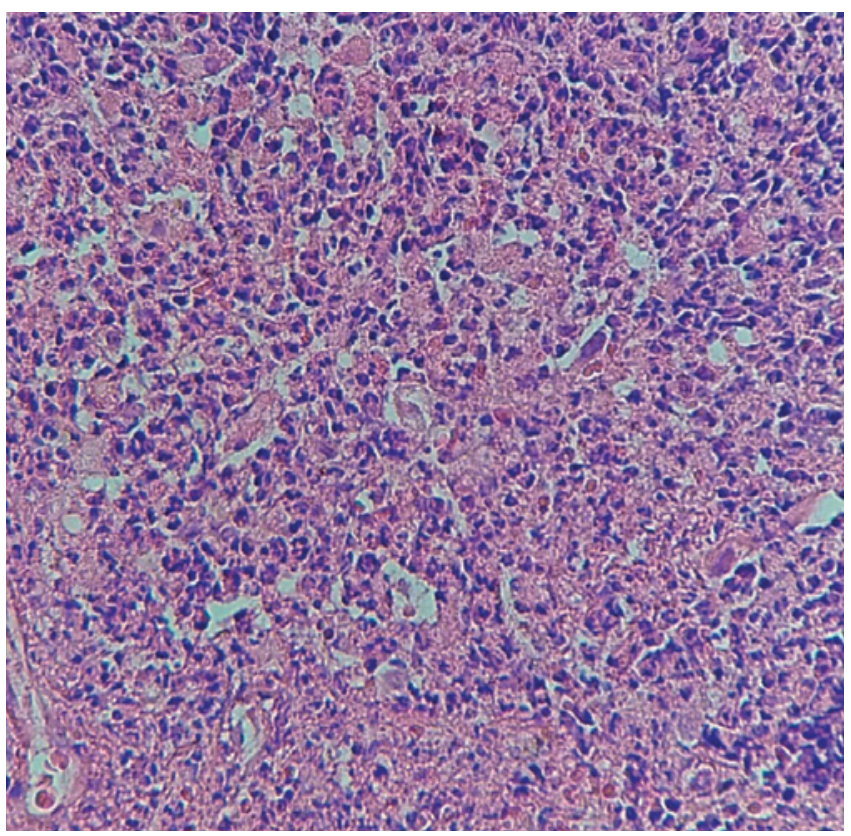

Figura 4 - Pormenor do infiltrado na derme profunda com infiltrado denso de neutrófilos envolvendo os anexos e vasos e extensa necrose do colágeno.

Durante 0 internamento, foi realizada antibioticoterapia com clindamicina endovenosa na dose de $600 \mathrm{mg} /$ dia associada a ciprofloxacina $400 \mathrm{mg} 2 \mathrm{x} /$ dia durante 6 dias, seguida de meropenem $500 \mathrm{mg} /$ dia (dose aumentada para $1 \mathrm{~g} /$ dia após 2 dias) e vancomicina $500 \mathrm{mg} 4$ x/dia, ambos por via endovenosa durante quinze dias. No décimo oitavo dia de internamento, iniciou-se prednisona oral, na dose total de $60 \mathrm{mg} /$ dia, observando-se regressão progressiva da lesão. A paciente apresentou remissão completa da lesão no primeiro mês e reduziu progressivamente a corticoterapia que foi suspensa ao fim de mais quatro semanas. Fez acompanhamento durante nove meses nos ambulatórios de Dermatologia e Hematologia sem recidiva do pioderma gangrenoso e sem modificação do quadro de HPN.

\section{DISCUSSÃO}

O pioderma gangrenoso é uma dermatose crónica, progressiva e rara que pode levar à necrose severa de tecidos, dor intensa e deformidades se não for adequadamente diagnosticada e tratada. ${ }^{1,9}$ Existem quatro variantes: ulcerada, bolhosa, vegetante e pustulosa. A forma mais frequente é a ulcerada, que se inicia com pápula ou nódulo e evolui rapidamente para lesões ulceradas e dolorosas. ${ }^{3}$ Os componentes que iniciam e mantêm as ulcerações não são claros, mas parecem ser multifatoriais, incluindo predisposição genética, agentes infecciosos, fenômenos paraneoplásicos ou paraimunes. $^{7}$

As lesões estão localizadas mais frequentemente nos membros inferiores, podendo ocorrer também nos membros superiores, tronco, cabeça e pescoço..$^{9,10} \mathrm{O}$ diagnóstico é feito por exclusão ao analisar o perfil clínico-laboratorial do paciente. Pelo fato desta doença não possuir evidências laboratoriais e histopatológicas características, tais exames apenas eliminam os diagnósticos diferenciais, ${ }^{3}$ nomeadamente vasculites sistêmicas, infecção cutânea e úlcera isquêmica. ${ }^{11}$

O tratamento é considerado controverso e não existem protocolos estabelecidos. As opções são: corticosteroides, imunossupressores e imunomoduladores. Prednisona em altas doses ( $1 \mathrm{mg} / \mathrm{kg} / \mathrm{dia}$ ) é o fármaco mais eficaz para o controle de manifestações do PG. ${ }^{11}$ Alguns estudos evidenciam que o tratamento de escolha para o PG são os corticoides orais ou endovenosos, a depender da gravidade do caso. Imunossupressores como ciclosporina, dapsona, azatioprina, clofazimina, talidomina, imunoglobulinas endovenosas e etanercept também têm sido utilizados. 3,12,13

Estudo realizado com pacientes portadores de dermatoses tratados com imunoglobulinas endovenosas (lg Ev) entre janeiro de 2004 e outubro de 2011 num Serviço de referência em Dermatologia de Portugal, evidenciou que paciente com PG, cujo quadro foi refratário após o tratamento convencional, obteve cicatrização completa da úlcera de membro inferior direito após 6 meses de tratamento. ${ }^{12}$

Num caso rara de associação a hepatite $C$ crónica o PG foi tratado eficazmente com etanercept, um anti-TNF-a, associado a prednisolona, com cicatrização completa das lesões em cerca de 3 semanas. ${ }^{13}$

Devido à eficácia do corticosteroide em dose elevada e de terapias imunossupressoras, anormalidades de imunidade têm sido especulado na patogénese. ${ }^{5}$ Mas vale ressaltar que existem estudos demonstrando a eficácia da oxigenoterapia hiperbárica como tratamento adjuvante do PG, a qual possibilita a redução das doses de corticoide empregadas, alívio significativo da dor e melhora da lesão. ${ }^{14,15}$

A HPN é uma síndrome constituída pela tríade anemia hemolítica, pancitopenia e trombose. Existem poucos dados acerca de sua epidemiologia, visto que é uma doença rara. Uma pesquisa feita pela organização médica PNH Education and Study Group (PESG - Grupo de Estudos e Educação sobre HPN) afirma que a prevalência de HPN é 19,9/1 milhão e a incidência é 1,3/1 milhão. ${ }^{16}$ Sua etiologia está associada a mutações do gene PIG-A de células-tronco hematopoiéticas, 


\section{Caso Clínico}

resultando na deficiência das proteínas reguladoras da ativação do sistema complemento (CD55 e CD59), ancoradas ao glicosilfosfatidilinositol (GPI) na membrana plasmática de várias células, nomeadamente nos eritrócitos. A ausência dessas proteínas leva à hemólise, provocada pela própria cascata do complemento desregulada. ${ }^{17-19}$ Além disso, acredita-se que a hemólise, a ativação descontrolada do sistema complemento e a ausência de outras proteínas normalmente ancoradas pela GPI contribuem para a trombogénese, ${ }^{19}$ estando a HPN entre as causas de trombofilia adquirida. ${ }^{20}$

Acredita-se que o acometimento cutâneo em pacientes com HPN, que é incomum, está relacionado com a sua tendência trombótica. ${ }^{10} \mathrm{~A}$ concomitância entre HPN e PG é rara, ${ }^{5}$ sendo apenas encontrada em três relatos na literatu-

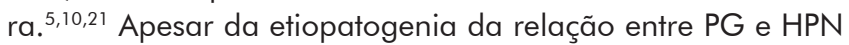
ser pouco elucidada, ${ }^{22}$ existem hipóteses que explicam a necrose das células epidérmicas no PG por uma diminuição da resistência dos queratinócitos e células endoteliais à lise celular provocada pelo sistema do complemento. ${ }^{5}$ Além do comprometimento eritrocitário da HPN, a expansão de um clone anormal hematopoiético é responsável por alterações, em proporção variável, de leucócitos e plaquetas em circulação, os quais também são susceptíveis de agressão pelo sistema complemento. ${ }^{10}$

Os neutrófilos de pacientes com HPN não possuem muitas moléculas de adesão celular ancoradas ao GPI em sua membrana, como a CD58, CD66c e CD67, o que pode estar relacionado ao acúmulo perivascular denso de neutrófilos na derme. Supõe-se também que os neutrófilos tenham anormalidades em suas fosfatases alcalinas, CD14 e redução da expressão do receptor Fcy IIIb (CD16) (cuja função é provocar a degranulação dos neutrófilos ao fazer ligação cruzada com anticorpos $\lg G), 5,10,23,24$ fazendo com que essas células respondam exacerbadamente a estímulos exógenos e se acumulem ao redor dos vasos. Ainda, a deficiência do receptor ativador de plasminogénio tipo uroquinase (CD87) pode ter um papel na trombose venosa ${ }^{16}$ e ulceração isquêmica que ocorre nas lesões de PG. ${ }^{5} \mathrm{~A}$ trombose é a complicação mais comum em pacientes com HPN, sendo o principal fator responsável pela mortalidade destes indivíduos. ${ }^{16}$

O diagnóstico de HPN pode ser atrasado pela variedade de achados clínicos da doença. Um diagnóstico precoce reduziria o risco de morbimortalidade. Após avaliação clínica, os achados laboratorias para diagnóstico de HPN consistem em três principais: marcadores laboratoriais de hemólise (hemograma, bilirrubina indireta, desidrogenase láctica, entre outros); demonstração da ausência das proteínas CD55 e CD59 ancoradas ao GPI em amostras de sangue periférico através da citometria de fluxo, o exame de excelência no diagnóstico de HPN; análise de medula óssea, não sendo esta última um indicador direto de HPN, mas necessária para saber se há deficiência de medula óssea ou não. ${ }^{16}$

O tratamento engloba três grupos: tratamentos de suporte (transfusão sanguínea em caso de anemia sintomática, ácido fólico e vitamina B12, suplementação oral de ferro, corticoides, anticoagulantes e imunossupressores), modificadores do curso da doença (eculizumab, um anticorpo monoclonal que bloqueia C5) e potencialmente curativos (transplante alogênico de medula óssea). ${ }^{16}$

Úlceras necróticas extensas tipo PG constituem uma das manifestações cutâneas dos estados de hipercoagulabilidade sanguínea (trombofilia). No caso de lesões com diagnóstico de PG que não curam, apesar do tratamento adequado, deve-se pesquisar trombofilias genéticas, principalmente se há história pessoal ou familiar de trombose. ${ }^{20}$

\section{CONCLUSÃO}

O pioderma gangrenoso ainda é uma doença de difícil diagnóstico, sendo necessários mais estudos para elucidar a sua patogênese. Acredita-se que a associação entre PG e HPN seja útil para nortear pesquisas futuras para entender a fisiopatogenia do PG e, consequentemente, melhorar o diagnóstico e tratamento dos pacientes acometidos por esta doença.

Conflitos de interesse: Os autores declaram não possuir conflitos de interesse.

Suporte financeiro: $O$ presente trabalho não foi suportado por nenhum subsídio ou bolsa.

Confidencialidade dos dados: Os autores declaram ter seguido os protocolos do seu centro de trabalho acerca da publicação dos dados de doentes.

Protecção de pessoas e animais: Os autores declaram que os procedimentos seguidos estavam de acordo com os regulamentos estabelecidos pelos responsáveis da Comissão de Investigação Clínica e Ética e de acordo com a Declaração de Helsínquia da Associação Médica Mundial

Conflicts of interest: The authors have no conflicts of interest to declare.

Financing Support: This work has not received any contribution, grant or scholarship.

Confidentiality of data: The authors declare that they have followed the protocols of their work center on the publication of data from patients.

Protection of human and animal subjects: The authors declare that the procedures followed were in accordance with the regulations of the relevant clinical research ethics committee and with those of the Code of Ethics of the World Medical Association (Declaration of Helsinki).

\section{REFERÊNCIAS}

1. Ruocco E, Sangiuliano S, Gravina AG, Miranda A, Nicoletti G. Pyoderma gangrenosum: an updated review. J Eur Acad Dermatol Venereol. 2009; 23:1008-17.

2. Konopka CL, Padulla GA, Ortiz MP, Beck AK, Bitencourt $M R$, Dalcin DC. Pioderma gangrenoso: um artigo de revisão. J Vasc Brasil. 2013; 12:25-33.

3. Santos M, Talhari C, Rabelo RF, Schettini APM, Chirano CA, Talhari S. Pioderma Gangrenoso: apresentação clínica de difícil diagnóstico. An Brasil Dermatol. 2011; 86:153-6.

4. Meyer TN. Pioderma gangrenoso: grave e mal conhecida complicação da cicatrização. Rev Brasil Cir Plást. 
$2006 ; 21: 120-4$

5. Matsubara K, Isoda K, Maeda Y, Mizutani H. Pyoderma gangrenosum associated with paroxysmal nocturnal hemoglobulinuria and monoclonal gammopathy. J Dermatol. 2002; 29:86-90.

6. Jockenhöfer F, Klode J, Kröger K, Roesch A, Al Ghazal $P$, Dissemond J. Patients with pyoderma gangrenosum analyses of the German DRG data from 2012. Int Wound J. $2016 ; 13: 951-6$.

7. Barbato MT, Bakos L, Masiero NCMS, Bolson P. Perfil clinicopatológico dos pacientes com pioderma gangrenoso do Hospital das Clínicas de Porto Alegre (RS) - Brasil (2000-2006). An Brasil Dermatol. 2008; 83:431-6.

8. Al Ghazal P, Herberger K, Schaller J, Strölin A, Hoff NP, Goerge T, et al. Associated factors and comorbidities in patients with pyoderma gangrenosum in Germany: a retrospective multicentric analysis in 259 patients. Orphanet J Rare Dis. 2013; 8:136.

9. Harris AJ, Regan P, Burge S. Early diagnosis of pyoderma gangrenosum is important to prevent disfigurement. BMJ. 1998; 316:52-3.

10. Goulden V, Bond L, Highet AS. Pyoderma gangrenosum associated with paroxysmal nocturnal haemoglobinuria. Clin Exp Dermatol. 1994;19:271-3.

11. Bittencourt MS, Soares LF, Lobato LS, Mançano AD, Leandro HS, Fonseca DM. Multiple cavitary pulmonary nodules in association with pyoderma gangrenosum: case report. An Brasil Dermatol. 2012; 87:301-4.

12. Araújo $C$, Fernandes JC, Duarte ML, Pereira T, Vieira AP, Brito C. Imunoglobulinas endovenosas em dermatologia - experiência clínica de 7 anos no hospital de braga. Rev Soc Port Dermatol Venereol. 2013; 71:34559.

13. Tavares E, Dores JA, Aranha J. Pioderma gangrenoso associado a hepatite c crónica tratado eficazmente com etanercept. Rev Soc Port Dermatol Venereol. 2012; 70:215-21.

14. Araújo FM, Kondo RN, Minelli L. Pyoderma gangrenosum: skin grafting and hyperbaric oxygen as adjuvants in the treatment of a deep and extensive ulcer. An Brasil Dermatol. 2013; 88:176-8.

15. Vieira WA, Barbosa LR, Martin LM. Oxigenoterapia hiperbárica como tratamento adjuvante do pioderma gangrenoso. An Brasil Dermatol. 201 1; 86:1193-6.

16. Sahin F, Akay O, Ayer M, Dal M, Ertop S, Ilhan O, et al. Pesg PNH diagnosis, follow-up and treatment guidelines. Am J Blood Res. 2016; 6:19-27.

17. Arruda $M M$, Rodrigues $C A$, Yamamoto $M$, Figueiredo MS. Hemoglobinúria paroxística noturna: da fisiopatologia ao tratamento. Rev Assoc Méd Brasil. 2010; 56:21421.

18. Brodsky RA. New insights into paroxysmal nocturnal hemoglobinuria. Hematology Am Soc Hematol Educ Program. 2006:24-8, 516.

19. Fraiman YS, Cuka N, Batista D, Vuica-Ross M, Moliterno AR. Development of paroxysmal nocturnal hemoglobinuria in CALR-positive myeloproliferative neoplasm. J Blood Med. 2016; 7:107-10.

20. Criado PR, Rivitti EA, Vasconcellos C, Valente NYS, Martins JEC. Manifestações cutâneas das trombofilias. An Brasil Dermatol. 2008; 83:491-506.

21. Holt PJ, Davies MG, Saunders KC, Nuki G. Pyoderma gangrenosum: clinical and laboratory findings in $15 \mathrm{pa}-$ tients with special reference to polyarthritis. Medicine. 1980; 59:1 14-33.

22. Batista MD, Fernandes RL, Rocha MA, Ikino JK, Pinheiro RF, Chauffaille ML, et al. Pioderma gangrenoso bolhoso e síndrome mielodisplásica. An Brasil Dermatol. 2006; 81:S309-S12.

23. Adu B, Jepsen MP, Gerds TA, Kyei-Baafour E, Christiansen $M$, Dodoo $D$, et al. Fc gamma receptor 3B (FCGR3B-c.233C >A-rs5030738) polymorphism modifies the protective effect of malaria specific antibodies in Ghanaian children. J Infect Dis. 2014; 209:285-9.

24. Hundt $M$, Schubert J, de Haas $M$, Zielinska-Skowronek $M$, Schmidt RE. The loss of Fc gamma Rlllb in paroxysmal nocturnal hemoglobinuria is functionally replaced by $\mathrm{Fc}$ gamma RIl. Blood. 1994; 83:3574-80. 